Article

\title{
A systematic review that evaluates the extent and quality of involving childhood abuse survivors in shaping, conducting and disseminating research in the UK
}

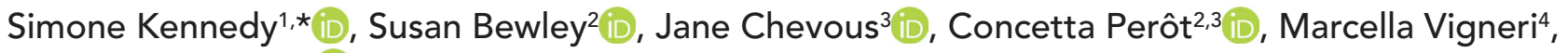 \\ Loraine J. Bacchus ${ }^{1}$ (D) \\ ${ }^{1}$ London School of Hygiene \& Tropical Medicine, UK \\ ${ }^{2}$ King's College London, UK \\ ${ }^{3}$ Survivors Voices, King's College London, UK \\ ${ }^{4}$ CEDIL, London School of Hygiene \& Tropical Medicine, UK \\ *Correspondence: simone.kennedy29@gmail.com
}

Submission date: 5 October 2020; Acceptance date: 18 October 2021; Publication date: 27 January 2022

\section{How to cite}

Kennedy, S., Bewley, S., Chevous, J., Perôt, C., Vigneri, M. and Bacchus, L.J. (2022) 'A systematic review that evaluates the extent and quality of involving childhood abuse survivors in shaping, conducting and disseminating research in the UK'. Research for All, 6 (1), 3, 1-25. DOI: https://doi.org/10.14324/RFA.06.1.03.

\section{Peer review}

This article has been peer-reviewed through the journal's standard double-blind peer review, where both the reviewers and authors are anonymised during review.

\section{Copyright}

2022, Simone Kennedy, Susan Bewley, Jane Chevous, Concetta Perôt, Marcella Vigneri and Loraine J. Bacchus. This is an open-access article distributed under the terms of the Creative Commons Attribution Licence (CC BY) 4.0 https://creativecommons.org/licenses/by/4.0/, which permits unrestricted use, distribution and reproduction in any medium, provided the original authors and source are credited • DOI: https://doi.org/10.14324/RFA.06.1.03.

\section{Open access}

Research for All is a peer-reviewed open-access journal.

\begin{abstract}
Despite a well-established understanding of the mental and physical health consequences associated with exposure to childhood abuse, the active voices of survivors are rarely present in shaping, conducting and disseminating research. To explore the extent and quality of involvement with adult survivors of childhood abuse in the UK, we performed a systematic review of research conducted 'with' or 'by' survivors, and analysed involvement against a new instrument, the Survivor Research Involvement Ladder, which was co-produced drawing from the principles of the Survivors Voices Charter. A search of relevant grey and peer-reviewed literature was conducted, which retrieved 662 sources after removing duplicates. Of these, 116 full-text articles on adult survivors of childhood abuse in the UK were subsequently assessed for involvement (beyond participation as 'subjects'), of which
\end{abstract}


only 15 (12.9 per cent) reported activities led, co-produced, advised or consulted on by survivors, and these were included in the review. From evaluations and analysis using the ladder, consumerist models were found to be the dominant form of involvement, with survivors filling advisory roles at isolated stages. Survivor-led research was scarce but emerged when survivor-researchers planned, conducted and disseminated their work. This review finds considerable opportunity for improvements in the level, quality and subsequent reporting of research activities involving survivors. The use of the instrument needs replication, validation and further field-testing.

Keywords childhood abuse survivors; meaningful involvement; experiential expertise; co-produced; evaluation tool; involvement ladder; systematic review

\section{Key messages}

- There is insufficient evidence of detailed reporting of quality research involvement practices with adult survivors of childhood abuse.

- Involvement activities in research with survivors are usually limited to an advisory capacity on isolated areas of research.

- A tool that evaluates survivor involvement can suggest and monitor areas for improvement.

\section{Introduction}

Since the UK's legislative imperative for patient and public involvement (PPI), documentation of PPI in research funding applications has increased (Elliott et al., 2017; Mathie et al., 2014). However, the lack of monitoring and insufficient clarity regarding implementation have left research teams and funders with the power to determine the scope and nature of PPI in practice (Paylor and McKevitt, 2019). Incongruent interpretations of PPI have led to varying degrees of involvement by design, topic and discipline (Mathie et al., 2014; Blackburn et al., 2018). There is a broad tendency for practices to be limited to a consumerist model of lay consultation on traditional scientific expertise (Paylor and McKevitt, 2019; Crompton, 2019; Beresford, 2013a). Motivated by pragmatic aims of improving the relevance and quality of research outputs, researchers have privileged perspectives from accessible, competent and socially prominent populations (Thompson et al., 2012; Beresford, 2013b). As a result, a diverse representation of voices has been limited (Green, 2016). By failing to address critical barriers to inclusion (Holland-Hart et al., 2019), research has neglected the experiences of marginalised groups and failed to capture voices that fall outside the normative discourse (Kalathil and Jones, 2016; Martin, 2008; Beresford, 2013b).

The voice of adult survivors of childhood abuse, hereafter referred to as 'survivors', has been critically absent from PPI in health planning and research. Despite a well-established understanding of the mental and physical health consequences associated with exposure to childhood abuse and adversity (Felitti et al., 1998; Carr et al., 2018), survivor narratives are minimally present in research, and insufficiently extend beyond traditional biomedical notions of 'treatment' and 'recovery' (MacGinley et al., 2019). Within an evidence base that primarily relies on psychiatric symptom scores to shape policy and service provision (Dorrepaal et al., 2014; Ehring et al., 2014), survivor narratives have been co-opted, dehumanised and pathologised in the current research agenda (Gibson and Morgan, 2013; Crepaz-Keay, 2016).

Although an emerging evidence base has begun to challenge the scientific research agenda by seeking survivor perspectives on therapeutic approaches to care and treatment (Anderson and Hiersteiner, 2008; Parry and Simpson, 2016; Wright and Gabriel, 2018; Chouliara et al., 2012), the predominant focus on traditional approaches undervalues experiential expertise in understanding the complexity and diversity in recovery experiences (Beckett et al., 2018). The need to harness the experiential knowledge of survivors in research has been further demonstrated by professionals adopting collaborative and patient-centred approaches due to growing scepticism of expert-informed 'best' practices (Munro and Randall, 2007). 
Despite research demonstrating that survivors similarly value collaborative working relationships in research (McClain and Amar, 2013), the extent to which these practices are integrated into UK research is not fully understood.

In other disciplines and country contexts, involvement has been found to challenge traditional ways of knowing and doing, thus giving rise to research outputs that are feasible, acceptable and contextually informed (Brett et al., 2014; Jennings et al., 2018; Thompson et al., 2014). Additionally, involvement has resulted in research adopting sensitive approaches and appropriate ethical protocols (Tembo et al., 2019; Lambert and Carr, 2018). Considering that there is a lack of well-defined ethical protocols for conducting research with survivors (Radhika et al., 2018), understanding if and how current involvement fosters ethical practices is of value to future research.

Beyond the benefits to knowledge production, involvement practices are critical to addressing potentially re-traumatising experiences of disempowerment and silencing when survivors are poorly engaged in research (Perôt et al., 2018). In New Zealand (Gibson and Morgan, 2013) and the United States (McClain and Amar, 2013), opportunities for survivors to have an active voice in research have been found to be a meaningful and emancipatory departure from enduring experiences of being silenced. Similarly, research conducted by survivors has been regarded as an empowering source of healing and self-development by researchers challenging one-dimensional and oppressive survivor stereotypes (Kara, 2013; LaFrance, 2017; Ramirez and Allison, 2018).

While the broad impacts have been reviewed extensively, and service user/mental health survivor involvement practices are well established in UK research (Sweeney et al., 2009; Faulkner, 2004), involvement practices need to be tailored and understood within the specific needs and contexts of this group (Tambuyzer et al., 2014). This systematic review aims to address this gap by establishing a benchmark of the number of peer-reviewed and grey literature studies with survivor involvement that exist to determine the scale of abuse survivors' voices in UK research. By piloting a new study evaluation tool developed by Survivors Voices (Chevous et al., 2019), this review has a complementary aim of evaluating the quality of involvement practices documented in these studies. Recommendations for conducting meaningful involvement practices with survivors will be offered following a discussion of the evaluation results, and suggested adaptations to the evaluation tool will be outlined to inform the development of revised versions. The research presented here is part of a larger systematic review conducted by the researchers that also sought to explore support, help-seeking and recovery experiences, perspectives and needs voiced by survivors. The methods and findings presented here only include information relevant to survivors' involvement evaluations.

\section{Approach}

In 2018, Survivors Voices, a national survivor-led organisation, developed Turning Pain into Power: A charter for engaging survivors in projects, research \& service development (Perôt et al., 2018). Authored by survivor activists, and developed in collaboration with King's College London, with support from Wellcome's Public Engagement Fund, the document provides guidance for individuals and organisations to facilitate meaningful engagement with survivors. Acknowledging that survivors are a significant population, yet largely silenced and excluded, Survivors Voices were motivated to transform the pain from their experiences into power to evoke change. The subsequent Survivors Charter regards engagement as possible and necessary. It acts when 'researchers, project staff and organisations are able to be empathic partners with survivors whose full experiences and voices are heard, heeded and allowed to have significant impact' (Perôt et al., 2018: 2). Cognisant of how the dynamics of abuse can be replicated by poor engagement, the Survivors Charter provides a set of principles to ensure that engagement does not evoke the power dynamics of past abuse, but demonstrates the opposite (see Table 1).

Following the Survivors Charter, the Survivor Research Involvement Ladder was developed to guide and assess the integration of principles for meaningful engagement into practice (Chevous et al., 2019). 
Table 1. Principles of the Survivors Charter for engaging abuse survivors (Perôt et al., 2018: 3)

Dynamics of abuse

Abuse is inherently unsafe. It

leaves a long legacy of fear.

People who are abusive

dominate and take away personal power.

Abuse is silencing.

Abuse is self-negating, destroys self-worth and damages wellbeing.

Abuse is hidden, and abusers often act with impunity.

Abuse restricts and arrests healthy growth, imprisoning people in physical, mental and emotional shackles.

Abuse is corrosive, restrictive and soul-destroying.

\section{Principles for meaningful engagement}

Safe Engagement should be in a safe environment that begins with providing attentive listening and connections that are warm, collaborative and relational, which recognises and minimises triggers and may include safety protocols.

Empowering Engagement should be collaborative, and must empower survivors to have control of decisions about their own involvement.

Amplifying the voices of survivors Engagement should help release and amplify survivors' voices, experiences and expertise.

Promoting self-care Engagement in research-activism can impact coping mechanisms - thus radical self-care should be normalised by example, as well as in organisational processes.

Accountable and transparent Engagement with survivors must have clear lines of communication and accountability. Processes and decision making should be relational, honest, real, transparent and open to feedback and dialogue.

Liberating Engagement should be liberating, dynamic, life-giving, and help survivors experience a sense of possibility and life beyond the aftermath of abuse. Engagement should be a totally voluntary process and easy to withdraw from at any point.

Creative and joyful Engagement should be a creative process. Good engagement focuses on positive experiences and strengths, as well as negative ones, and can increase capacity for joy, creativity and imagination.

The development of the tool was informed by Arnstein's (1969) Ladder of Citizen Participation and an adapted version by Hart (1992). Of relevance to the principles of good involvement in the Survivors Charter, Arnstein (1969) conceptualised involvement as the degree of empowerment, or the amount of power transferred from the haves to the have-nots. From tokenism to citizen control, Arnstein's (1969) rungs represent a hierarchy of involvement, with each stepwise level corresponding with the degree of power that individuals have in shaping projects. Tokenistic practices, which occupy rungs between non-participation and citizen control, allow individuals to have a voice and be heard, but their views are not necessarily implicated in decision making. These practices critically lack the components of good engagement in the Survivors Charter, particularly that survivors' voices should be 'heeded and allowed to have a significant impact' (Perôt et al., 2018: 2). Absence of these elements in involvement can be re-traumatising experiences by inadvertently replicating the silencing, disempowering and exclusionary dynamics of abuse (Perôt et al., 2018). Drawing from the elements of Arnstein's (1969) work, the Survivor Research Involvement Ladder was developed as a practical tool for evaluating the level of empowerment implicated in their involvement roles, and it will be further detailed in the subsequent methods section. Last, this review is a product of survivor involvement, as it was collaboratively designed, conducted and disseminated with members of Survivors Voices.

\section{Methods}

\section{Eligibility criteria}

To benchmark the number of studies involving adult survivors of childhood abuse in the UK, studies were only included for subsequent involvement evaluations if they met the initial criteria relating to the population of interest, location and publication status. 
Studies included adult participants, 18 years or older, whose abuse occurred under the age of 18 . No specifications were placed on the types of childhood abuse, and they included:

- physical, sexual and emotional abuse, neglect and witnessing trauma

- abuse perpetrated by an individual, group or institution

- abuse perpetrated by adults or children

- abuse where the survivor's relationship to the perpetrator is familial or non-familial.

Acknowledging the value of grey literature in PPI research (Brett et al., 2014), studies were not excluded based on their publication status. However, within grey literature sources, an emphasis was placed on locating studies widely accessible to the survivor and non-survivor populations, thus excluding dissertations. Studies were excluded if they were not based on primary research, but no limits were placed on the methodological design.

Studies that met the previous eligibility criteria were subsequently assessed for survivor involvement and evaluated using the Survivor Research Involvement Ladder at full-text screening. Studies were considered eligible if they explicitly stated that survivors' roles in research extended beyond being participants or subjects, by consulting, advising, co-producing or leading research activities at any point.

\section{Search strategy}

While piloting the search strategy, studies were found to use a wide variety of terminology in this subject area, and the use of search terms for certain concepts risked missing relevant studies. Decisively, the search strategy was designed to be broad and limited to three key concepts: 'child abuse', 'adult survivor' and 'UK'. Studies were found to omit terms relevant to involvement practices in the title and abstract; therefore, involvement was assessed using the ladder at full-text screening.

A variety of relevant search terms within the three concepts were combined with the appropriate use of Boolean operators (see Table 2). When supported by database search formats, proximity commands were used to capture variability in terminology and ordering within the key concepts of 'child abuse' and 'adult survivors'. Furthermore, relevant database-specific Medical Subject Heading (MeSH) terms were combined with each of the key concepts.

The review consisted of a comprehensive search of relevant studies by systematically searching academic databases, and by systematically searching relevant databases and alternative information sources for grey literature.

\section{Search of academic databases}

A formal search of peer-reviewed studies was conducted using six academic databases: MEDLINE, CINAHL, Scopus, PsycINFO, International Bibliography of Social Science (IBSS) and Web of Science. The selection of databases aimed to include comprehensive multidisciplinary health databases (MEDLINE, CINAHL and Scopus), as well as databases specific to social sciences and psychology (PsycINFO, IBSS and Web of Science).

\section{Search of grey literature information sources}

Complementary strategies for sourcing grey literature reports were adapted from prior guidelines (Godin et al., 2015; Adams et al., 2016) to maximise the likelihood of collecting all relevant sources. The search strategy comprised three approaches and was completed as follows: (1) search terms were applied to two grey literature-specific databases, Social Care Online and Social Policy and Practice; (2) advanced Google searches were then used to locate online registries and websites of relevant organisations and agencies; and (3) reports were sourced from members of Survivors Voices, who have a high degree of awareness and exposure to research in the field. 
Table 2. Primary search terms used (adapted from MEDLINE without MeSH terms)

\begin{tabular}{|c|c|c|c|c|}
\hline Child & Abuse & Adult & Survivor & UK \\
\hline $\begin{array}{l}\text { (child* or infan* or } \\
\text { teenage* or teens } \\
\text { adolescen* or youth* } \\
\text { or juvenile* or kid* } \\
\text { or minor* or girl* or } \\
\text { boy* or p?diatric* or } \\
\text { preschool* or "pre- } \\
\text { school* toddler*" or } \\
\text { newborn* or "early } \\
\text { year" or "school } \\
\text { student*" or pupil* } \\
\text { or step-child* or } \\
\text { stepchild* or baby or } \\
\text { babies or youth* or } \\
\text { "school age*") } \\
\text { OR } \\
\text { (elementary or primary } \\
\text { or nursery or secondary } \\
\text { or high or middle) adj2 } \\
\text { (school*) } \\
\text { OR } \\
\text { (first or second or third } \\
\text { or fourth or fifth or sixth } \\
\text { or seventh or eighth } \\
\text { or ninth or tenth or } \\
\text { eleventh or twelfth) } \\
\text { adj2 (grade*) }\end{array}$ & $\begin{array}{l}\text { (abus* or trauma* or } \\
\text { neglect* or maltreat* or } \\
\text { mistreat* or violen* or } \\
\text { exploit* or incest* or punish* } \\
\text { or abduct* or fondling* or } \\
\text { abandon* or moleste* or } \\
\text { molestation or assault* or } \\
\text { harm* or traffick* or bully* or } \\
\text { bullie* or harass* or deprive* } \\
\text { or battered or rape or raped } \\
\text { or advers* or incest*) } \\
\text { OR } \\
\text { (lack* or deprive* or } \\
\text { insufficient* or inadequat* or } \\
\text { poor) adj2 (care or affection } \\
\text { or attention or supervis* or } \\
\text { contact* or attachment) } \\
\text { OR } \\
\text { (expose* or witness*) adj3 } \\
\text { (violen*) } \\
\text { OR } \\
\text { (unwanted or unlawful } \\
\text { or coerc* or forced or } \\
\text { non?consensual or offenc*) } \\
\text { adj2 (touch* or sex* or } \\
\text { intercourse or intimacy or } \\
\text { penetration or groom* or } \\
\text { rape or raped or p?edophil* } \\
\text { or prostitute* or pimp) } \\
\text { OR } \\
\text { (adverse or trauma*) adj2 } \\
\text { (childhood* or "childhood } \\
\text { experience*" or events or } \\
\text { upbringing* or family or } \\
\text { families or household* or } \\
\text { "home environment*" or } \\
\text { communit* or media or } \\
\text { school*) }\end{array}$ & $\begin{array}{l}\text { (adult* or m?n } \\
\text { or wom?n or } \\
\text { female* or } \\
\text { male*) }\end{array}$ & $\begin{array}{l}\text { (victim* or } \\
\text { survivor* or } \\
\text { histor* or } \\
\text { experienced }\end{array}$ & $\begin{array}{l}\text { ("United } \\
\text { Kingdom" } \\
\text { or England } \\
\text { or "Great } \\
\text { Britain" or } \\
\text { "Northern } \\
\text { Ireland" or } \\
\text { Scotland or } \\
\text { Wales) }\end{array}$ \\
\hline
\end{tabular}

\section{Study selection}

Screening for inclusion was facilitated by uploading all search results into Covidence online software. After the removal of duplicates, titles and abstracts were screened by the principal researcher. Full-text versions of all articles were then accessed and uploaded to Covidence. If full-text articles were not accessible to researchers through conventional means, authors or affiliated organisations were contacted by two researchers through emails detailing the purpose of the review and requesting electronic copies of research. Full-text versions of studies were assessed by the principal researcher according to the eligibility criteria, and exclusion was classified according to unmet criteria. Uncertainty about inclusion was resolved by discussing eligibility with the research team until consensus was met. 


\section{Quality appraisal}

The review utilised the Mixed Methods Appraisal Tool (MMAT), Version 2018 (Hong et al., 2018) to appraise the quality of studies, as it is appropriate for addressing methodological variability and permits assessments across diverse study designs. All relevant studies were included, regardless of methodological quality, as the research sought to include grey literature sources that may not abide by academic reporting standards. Following guidelines from MMAT, Version 2011 (Pluye et al., 2011), scores were calculated as a proportion of the relevant criteria met and tabulated alongside results from the Survivor Research Involvement Ladder to facilitate comparative evaluations. The potential for low-quality reporting practices across grey literature sources informed subsequent analysis assessing the influence of methodological quality on assessments of survivor involvement.

\section{Survivor Research Involvement Ladder assessment tool}

Constituting the main rungs of the ladder, and informing subsequent scoring of studies, are five roles that survivors may adopt in the research process (Chevous et al., 2019), each with varying degrees of correlation with the principles of the Survivors Charter (see Table 3). At opposite ends of the spectrum,

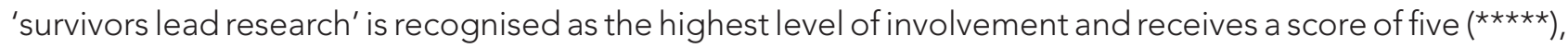
and 'survivors are only subjects or participants of the research' is the lowest level of involvement and is recognised with a score of one $\left({ }^{*}\right)$. Evaluations under key research activities were assisted by indicators of what the five roles may look like in practice. While the matrix initially recognised six key areas, adaptations were made during preliminary scoring to remove the criteria for assessing the availability of the report from the 'Dissemination' column. With the addition of a seventh column for 'Accessibility', involvement under this area was allowed to be appropriately evaluated.

Scoring is based on the values of Survivors Voices and the belief that involvement is 'possible, ethical ... and essential to getting the best results, that fully and accurately represent the experiences, views, wishes and feelings of survivors - the survivors' voices' (Perôt et al., 2018: 5). Assessments thus reflect this, by distinguishing the best research as survivor-led from start to finish. However, scoring requires explicit reporting of the research process pertaining to survivor involvement in order to make clear judgements. Therefore, the results reflect what is documented, which may or may not be the full extent of involvement in practice. While scoring may not reflect the true nature and scope of involvement, the tool places value on transparent and explicit reporting, as it frames involvement as a priority and recognises the value added when the platform is shared with survivors.

\section{Data extraction and analysis}

Descriptive characteristics of the included studies were extracted and tabulated to facilitate crosscomparisons. Piloting the ladder in this review, involvement was evaluated during study selection, then revised, refined and finalised once inclusion was decided. Total scores were calculated for each study to assess similarities and differences in overall involvement between studies, and scores under key areas were calculated and tabulated. To finalise involvement evaluations, the research team discussed scores until consensus, and adapted accordingly.

The subsequent element of analysis involved translating the data using thematic analysis. Although thematic analysis is typically used when analysing primary qualitative research, it can be used to systematically identify relevant information across multiple studies (Mays et al., 2005). An a priori thematic framework was developed from the seven key research areas for involvement (Chevous et al., 2019). Fulltext copies of studies were uploaded into NVivo 12 software, and evidence of survivors' involvement was coded descriptively according to the research activity in which it was documented. Descriptive codes documenting the level of involvement under the seven research areas were assisted using role-specific indicators (Chevous et al., 2019), and were then reviewed, refined and reorganised to develop analytical themes based on recurrent and prominent findings within and between studies. 


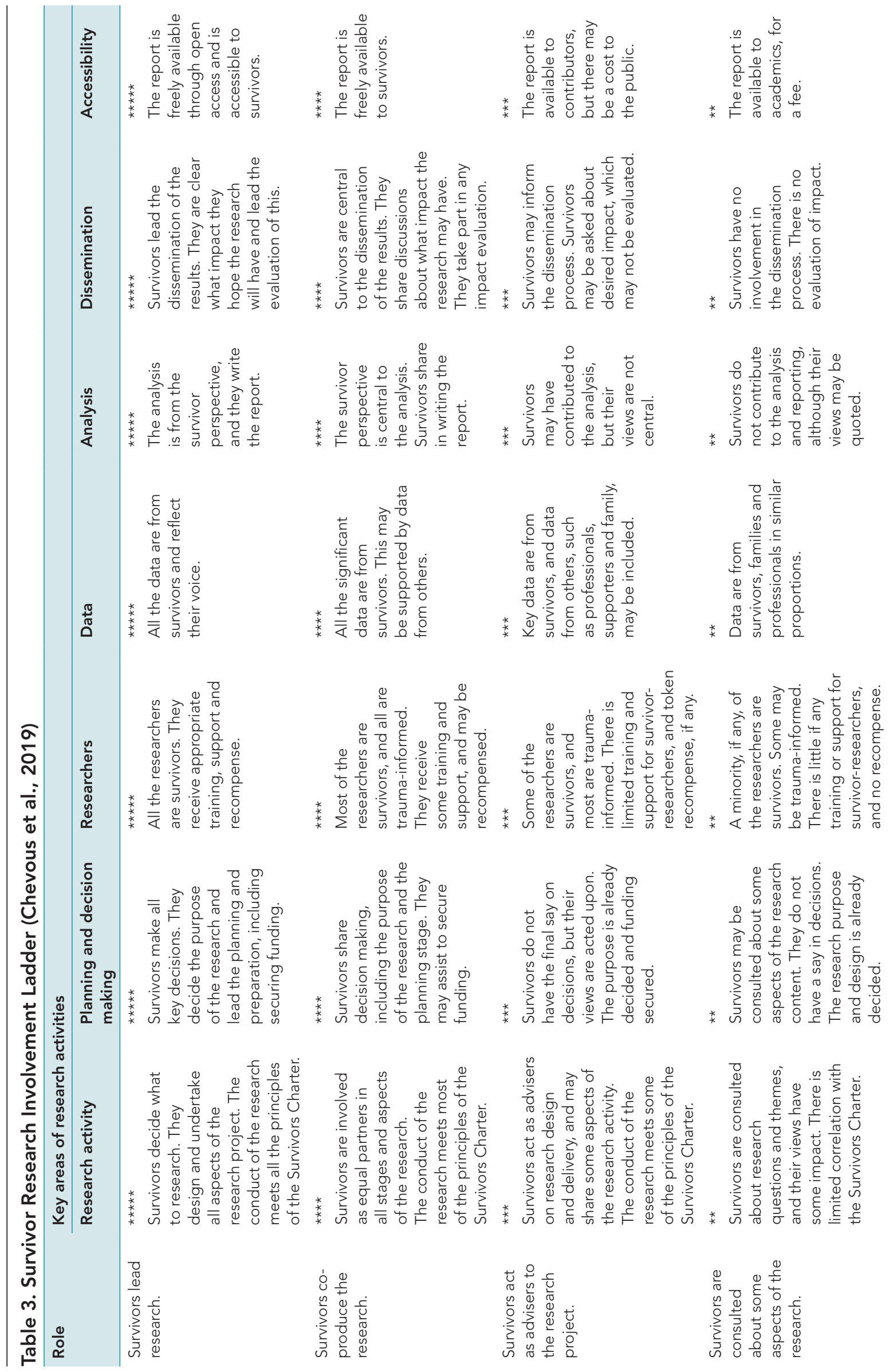




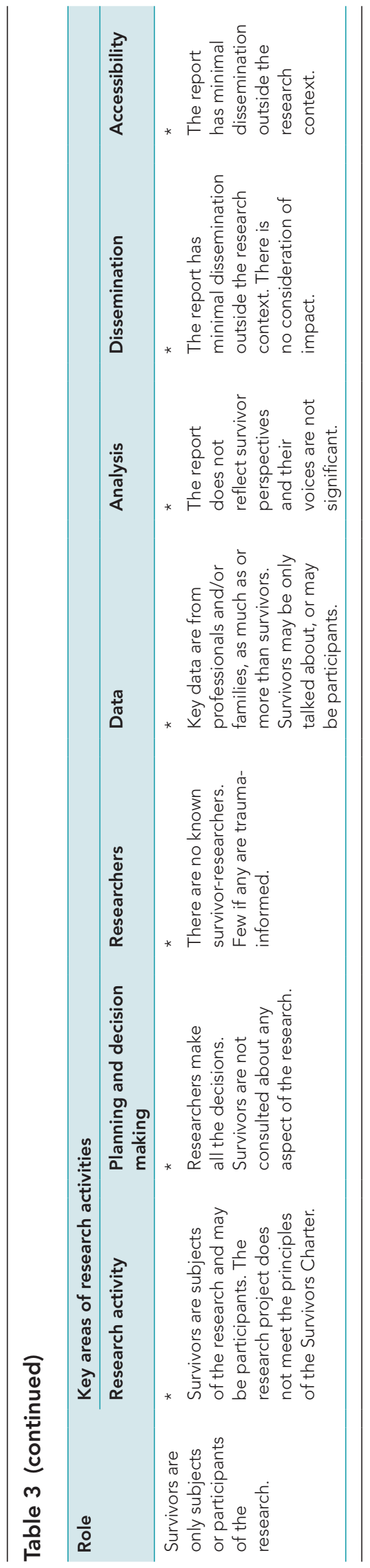




\section{Findings}

\section{Search results}

The database search retrieved 967 results, and an additional 45 grey literature sources were retrieved using the non-database search strategy (see Figure 1). After the removal of duplicates, 662 sources were screened by title and abstract, and 448 were subsequently excluded. There were 34 sources retrieved from the non-database search strategy, which were not captured by database searches and were included at the full-text screening phase. With the addition of 180 sources from database searches, a total of 214 full-text sources were read and assessed for eligibility.

Figure 1. PRISMA flow diagram of study selection process

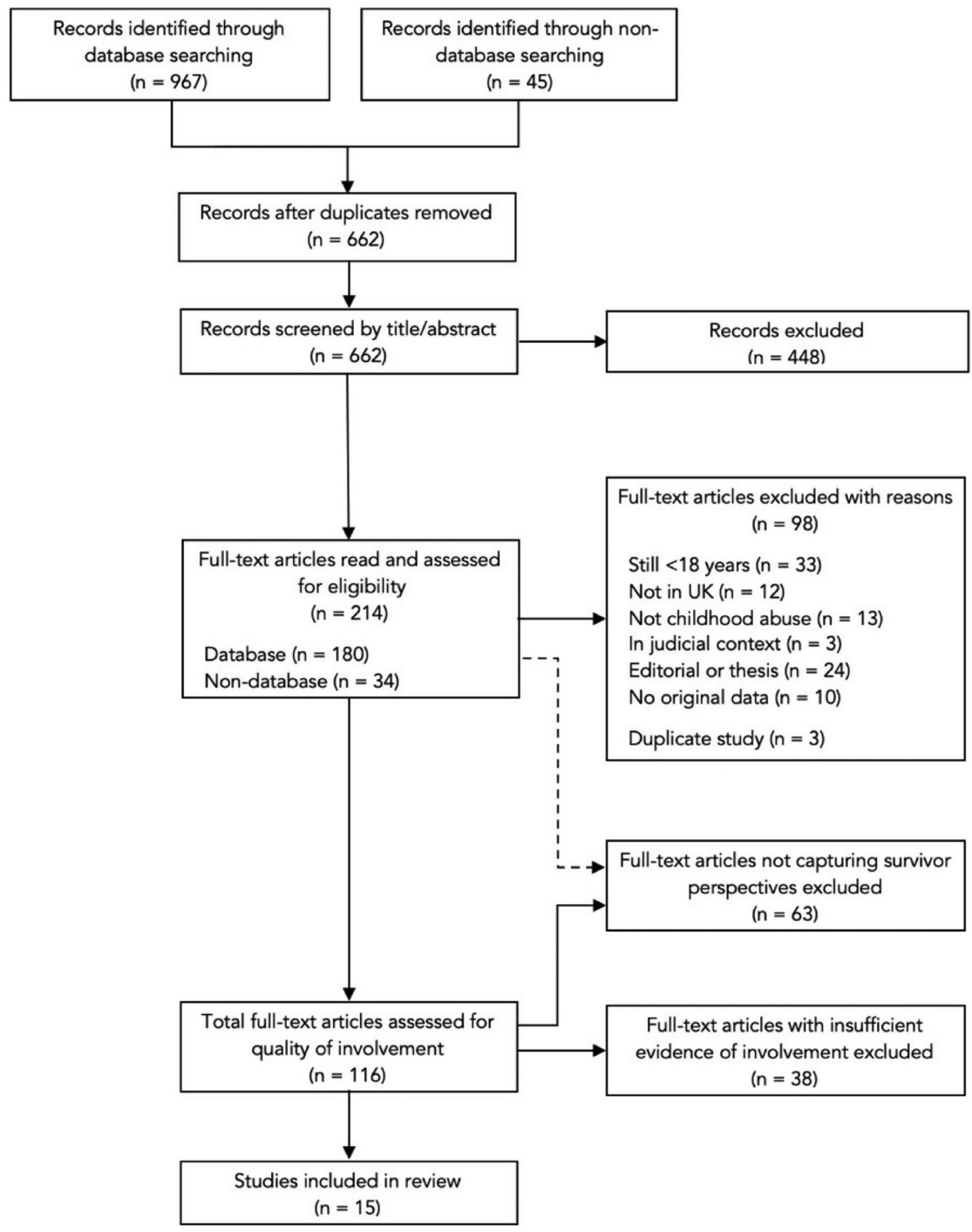


Following full-text assessments, 18 studies were eligible for inclusion. However, after applying classification criteria to evaluate study overlap (Urbanowicz and Reinke, 2018; Von Elm et al., 2004; Lefebvre et al., 2011), three pairs of studies were found to demonstrate a complete overlap in ideas and data. Following guidelines (Von Elm et al., 2004), the earlier source was regarded as the main article, and the study published at a later date was categorised as the corresponding article. When applicable, corresponding articles were used for gathering additional information on methods to assist with survivor involvement evaluation. However, corresponding articles were not appraised for quality. In total, 15 unique studies were selected for the review.

\section{Extent of survivor involvement in research}

Of the 214 studies screened by full text, 98 studies were ineligible due to the sample, setting, study type, publication status and evidence of study duplication. The remaining studies were assessed for: (1) capturing experiences, perspectives and needs voiced by survivors; and (2) evidence of survivor involvement beyond roles as subjects and participants (see Table 4). Of the 116 studies screened, 51 sources included survivors as participants but did not seek experiences or perspectives, and 12 sought service provider perspectives. Following involvement assessments, these 63 studies not seeking survivor experiences and perspectives were also found to not report involvement with survivors.

The research found that 53 of the 116 studies screened by full text captured the help-seeking, support and recovery perspectives and experiences of adult survivors of childhood abuse in the UK. Despite meeting the additional eligibility criteria, 38 were excluded due to insufficient evidence of survivor involvement beyond roles as subjects or participants. Therefore, of the 116 studies done 'for', 'about' or 'to' survivors, 12.9 per cent (15) were conducted 'with' or 'by' survivors, and of the 53 studies seeking survivor experiences and perspectives, only 28.3 per cent (15) explicitly reported involvement with survivors.

\section{Descriptions of the selected studies}

Among the 15 included studies, 8 were from grey literature sources and 7 were published in peerreviewed journals (see Table 5). Four of the studies used mixed methods (MM) designs and the remaining studies were purely qualitative (QUAL). Qualitative components of the included studies primarily used narrative approaches, with interviews and written accounts being the dominant form of data collection. Eleven of the included studies recruited participants through voluntary organisations and agencies, with participants having previous contact with service provision. Of the 15 studies, 10 had a predominate focus on child sexual abuse (CSA), one on ritual abuse (RA), and one on institutional child abuse (ICA). The remaining three studies looked broadly at all traumatic or adverse childhood experiences (ACE), which also included neglect, child emotional abuse (CEA) and child physical abuse (CPA). Among the studies presenting demographic information of participants, there was an observed lack of diversity in terms of gender and ethnicity, with most participants being White and female.

\begin{tabular}{|c|c|c|c|c|}
\hline \multirow{2}{*}{$\begin{array}{l}\text { Seeking direct perspectives } \\
\text { of participating survivors }\end{array}$} & \multicolumn{2}{|c|}{ Survivor involvement } & \multirow[t]{2}{*}{ Total } & \multirow{2}{*}{$\begin{array}{l}\text { \% of survivor } \\
\text { involvement }\end{array}$} \\
\hline & Yes & No & & \\
\hline Yes & 15 & 38 & 53 & 28.3 \\
\hline No & 0 & 63 & 63 & 0 \\
\hline Total & 15 & 101 & 116 & 12.9 \\
\hline
\end{tabular}




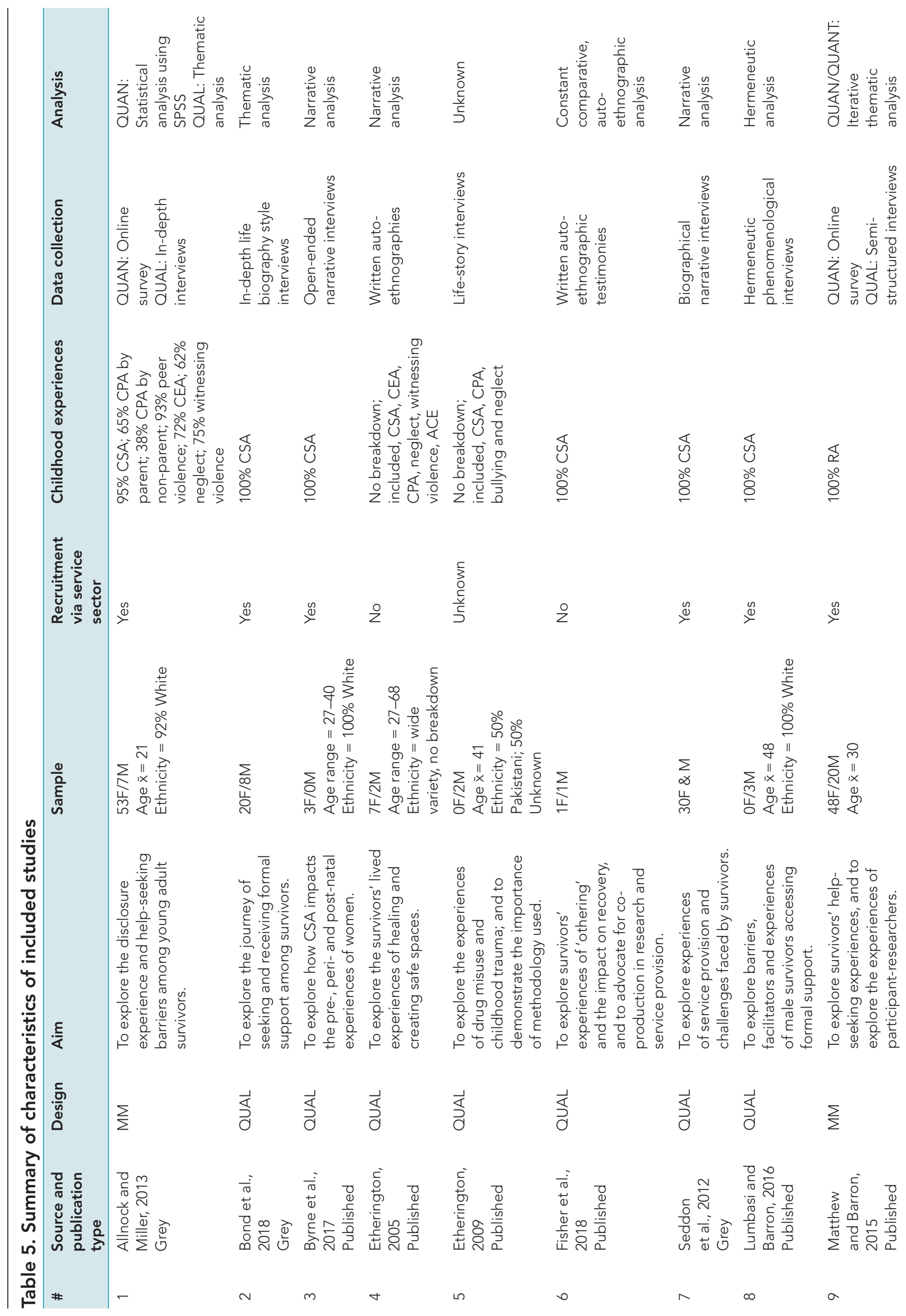




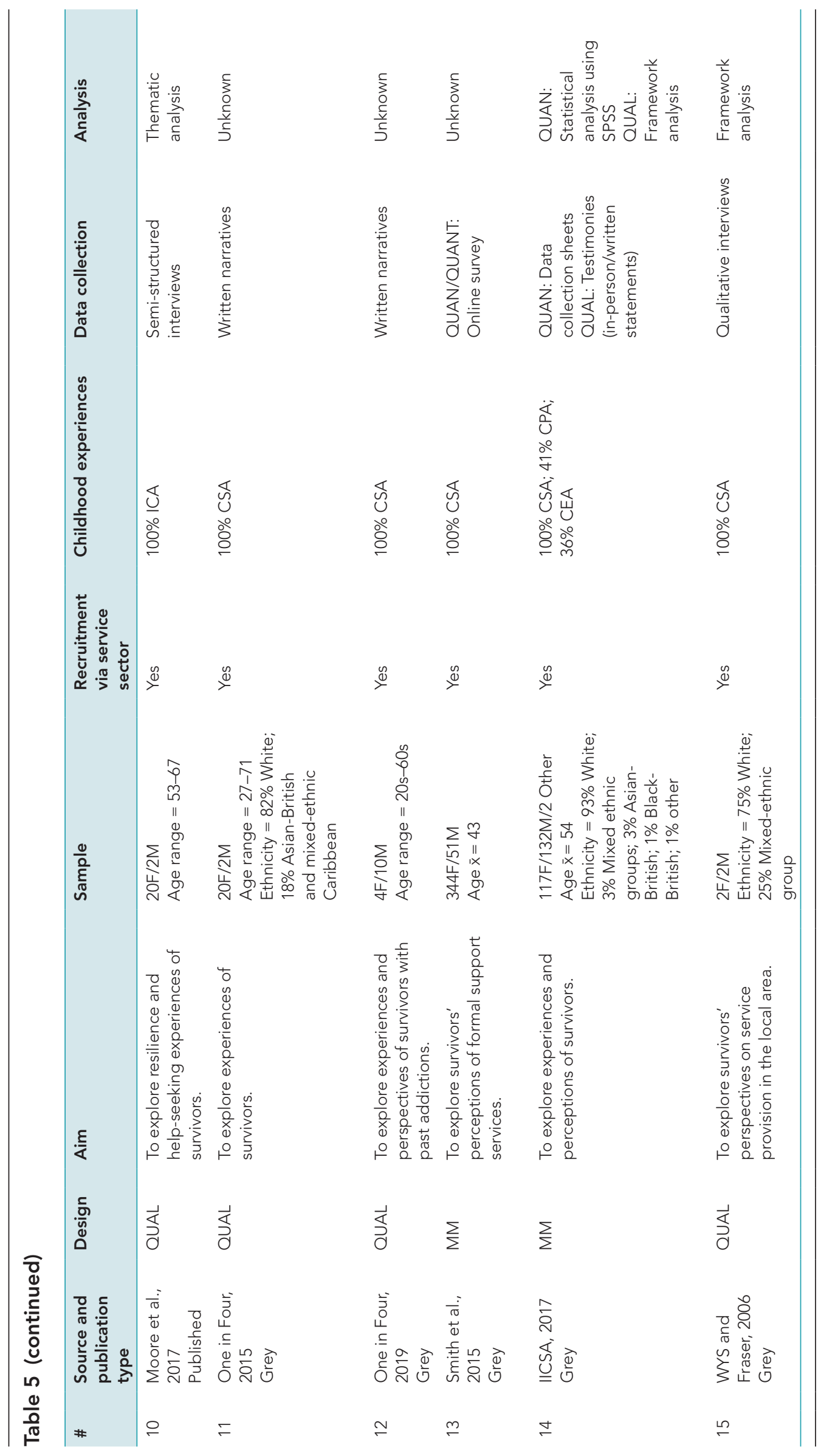




\section{Appraisal of methodological quality}

Quality appraisals using the MMAT resulted in the 11 qualitative studies receiving judgements of strong methodological quality (see Table 6). However, some grey literature sources provided insufficient descriptions of methodology, thus limiting comprehensive judgements. The four mixed methods studies had lower methodological quality, due to ill-defined target populations, inadequate sampling strategies or unsatisfactory methodological detail (Allnock and Miller, 2013; Matthew and Barron, 2015; Smith et al., 2015; IICSA, 2017).

When involvement with survivors was observed in quantitative (QUAN) components of mixed methods studies, this did not result in improved judgements of methodological quality. Therefore, despite mixed methods research demonstrating low quantitative quality, there was no observed influence on assessments of survivor involvement. Conversely, involvement with participating survivors during analysis was beneficial for assessments of methodological quality under qualitative components, as these activities were judged to improve the validity and transparency of the analysis and findings. However, when involvement was isolated to analysis, they added value to the appraisal of qualitative components but had a lesser effect on overall survivor involvement evaluations.

\section{Quality of survivor involvement in research}

Of the UK research literature on the experiences and perspectives of adult survivors of childhood abuse, only 12.9 per cent (15/116) of studies were found to demonstrate any survivor involvement. Of these 15 studies, the average overall score from the Survivor Research Involvement Ladder was 25.1 out of a total possible score of 35 , with a standard deviation of 5.4 (see Table 6). This value was positively skewed by distinctly high scores from four studies conducted by survivors (Etherington, 2005, 2009; Matthew and Barron, 2015; Fisher et al., 2018). After removing these four studies, the average score was 22, with a standard deviation of 1.5. Two authors, each with two studies included (Etherington, 2005, 2009; One in Four, 2015, 2019), were found to adopt similar involvement practices, which was reflected in subsequent scoring. To avoid exaggerating the impact of these two high-scoring authors, an analysis was performed to weigh the two sets of studies as one for each author, which produced an overall mean of 24.5, with a standard deviation of 5.2 .

While overall scores were found to be useful for broadly capturing similarities and differences in involvement across studies, the following summaries are presented based on the headings of the ladder to facilitate transparency and to provide a critical overview of involvement within key areas of the included research.

\section{Research activity}

Of the 15 studies, 6 received suboptimal scores as survivors adopted the role of consultants on isolated areas of the research and were minimally aligned with the principles of the Survivors Charter (Perôt et al., 2018). Where information was provided, studies sought consultation on ethical guidelines and protocols to improve safety (Allnock and Miller, 2013), on data collection methods to ensure relevance and acceptability (Smith et al., 2015; Bond et al., 2018), or on analysis to improve the quality and credibility (Byrne et al., 2017; Lumbasi and Barron, 2016). Engagement with survivor advisory groups at multiple stages of the research process was less frequently observed but demonstrated a more genuine form of collaboration in two instances (IICSA, 2017; Seddon et al., 2012). Although these studies showed a high degree of potential for meeting the principles of the Survivors Charter, a lack of sufficient information limited assessment scores.

Studies that provided participating survivors with opportunities to have an active role in research activities were found to demonstrate greater alignment with some of the Survivors Charter principles. The use of flexible and survivor-led narrative approaches to data collection was found to be empowering and liberating, and amplified the voices of participating survivors (Matthew and Barron, 2015; IICSA, 


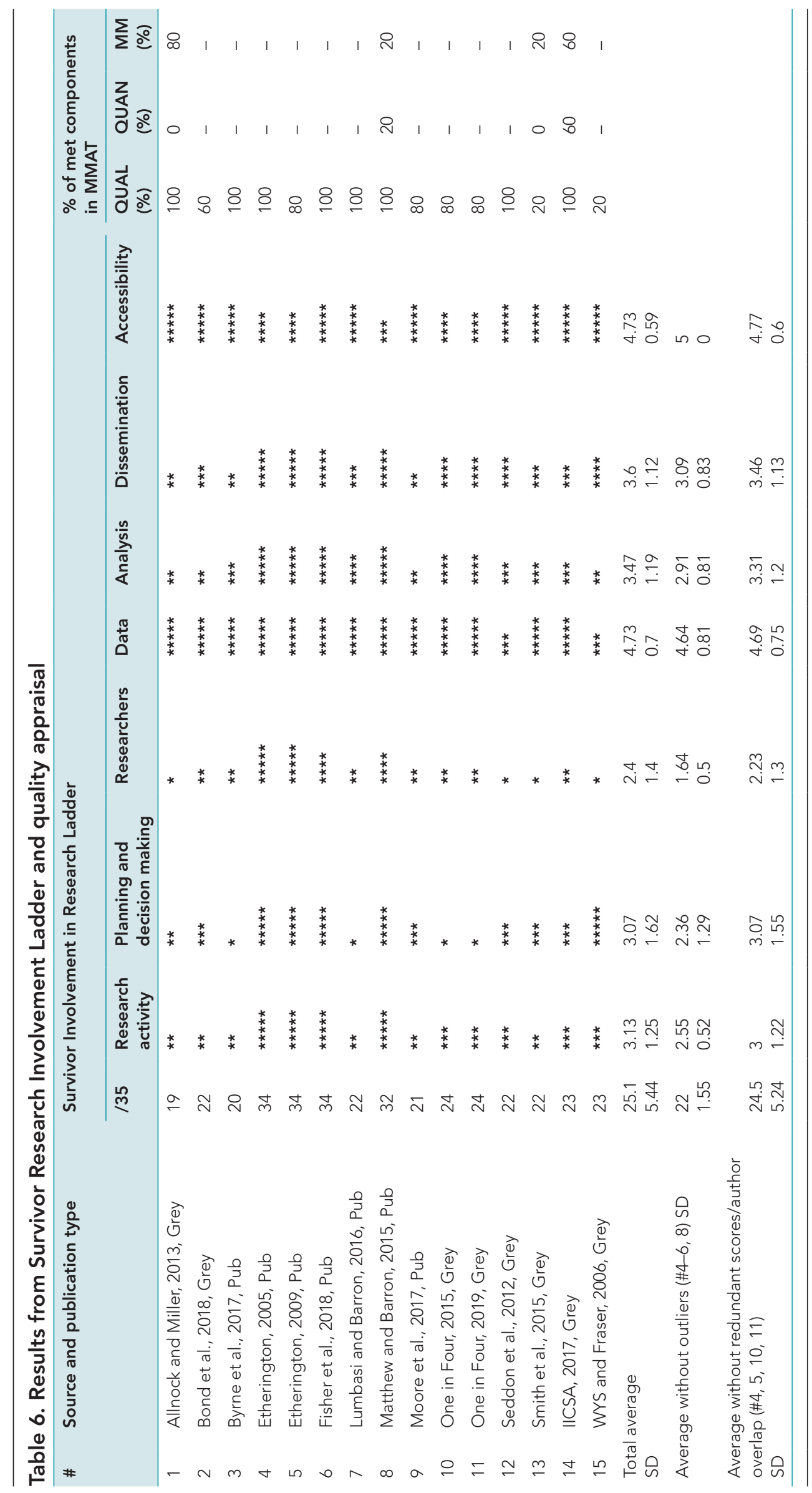


2017; Seddon et al., 2012; Lumbasi and Barron, 2016; One in Four, 2015, 2019; Etherington, 2005, 2009). Additionally, data collection through survivor-led writings was found to demonstrate non-restrictive and creative forms of engagement (IICSA, 2017; One in Four, 2015, 2019; Etherington, 2005). Soliciting input and engaging in dialogue at the analysis stage provided a space for survivors to contribute their expertise to the research (Matthew and Barron, 2015; Lumbasi and Barron, 2016). However, scoring was lowered if engagement with participating survivors was reported at isolated stages of data collection and analysis.

Last, the core values of the Survivors Charter were frequently observed in research conducted by survivor-researchers, as they were found to prioritise facilitating authentic and equal working relationships with survivors in practice (Etherington, 2005, 2009; Fisher et al., 2018; Matthew and Barron, 2015).

\section{Planning and decision making}

With the exception of survivor-led research, survivors did not fill roles at the planning stage, and the purpose of the research was decided before involvement commenced. The process of applying for and securing funding was not well documented and provided insufficient information to suggest survivors were involved. Some studies sought input from survivors on the design (Allnock and Miller, 2013; Smith et al., 2015; IICSA, 2017; Bond et al., 2018; Moore et al., 2017; WYS and Fraser, 2006); however, studies rarely elaborated on the consultative process and provided unsatisfactory detail about the degree of influence that these inputs had.

When survivors were not involved in the planning process, researchers made decisions to ensure survivors had a high degree of control over their voices as participants (Byrne et al., 2017; Lumbasi and Barron, 2016; One in Four, 2015, 2019). While survivor inputs did not actively guide these decisions, they did demonstrate how planning can lead to benefits of high-quality engagement at later stages. All studies by survivor-researchers were given full scores, and an additional study by WYS and Fraser (2006) received full scores as the study was commissioned by a survivor-founded and-led organisation.

\section{Researchers}

Of the 15 studies, 4 received high scores due to being conducted entirely or in part by survivor-researchers; however, 2 of these studies were authored by the same researcher (Etherington, 2005, 2009). The remaining studies received suboptimal scoring, with some additional recognition given if researchers were traumainformed (IICSA, 2017; Bond et al., 2018; Byrne et al., 2017; Lumbasi and Barron, 2016; Moore et al., 2017), and if training was given to survivors (One in Four, 2015, 2019).

\section{Data}

The data from the selected studies were frequently sourced entirely from survivors and received complete scores under this criterion. Conversely, studies that also included data from service providers received lower scores, despite survivors still representing the majority of the data (Seddon et al., 2012; WYS and Fraser, 2006). These assessments are informed by the potential for survivors' accounts and experiences to be co-opted or questioned by data sourced from service providers, family members or others talking about survivors. The ladder gives preference to research that directly seeks data about survivors from those with lived experience, and which allows their voices to stand alone as credible and reliable.

\section{Analysis}

Under the analysis criteria, low scores were allocated if results were supplemented by quotations, even when there was no evidence of involvement at this stage. Four studies did not demonstrate sufficient evidence of survivors' voices throughout the findings, three of which were grey literature reports. Two grey literature reports by One in Four $(2015,2019)$ met the criteria for higher scores by presenting raw accounts in full text, and by keeping survivors' voices central to the findings. 
Research that sought advice from survivors as members of project reference groups received adequate scores if these groups assisted with refining coding schemes (IICSA, 2017) or provided feedback on preliminary analysis (Seddon et al., 2012). However, these practices were observed to be minimally collaborative, and survivors did not have a central role in the analysis. Conversely, when participating survivors had an active role in analysis, it was found to blur the power differentials between researchers and participants through ongoing dialogue guided by their experiential expertise (Matthew and Barron, 2015; Lumbasi and Barron, 2016; Etherington, 2009; Fisher et al., 2018). As a result, involvement during analysis not only strengthened the credibility of the research, but also facilitated empowerment by allowing survivors to maintain control over the nature of participation.

\section{Dissemination}

There was insufficient information detailing the dissemination process in both published and grey literature studies. Seven studies provided information about the dissemination process, including survivors interpreting the results (IICSA, 2017; Smith et al., 2015; Bond et al., 2018), reviewing and shaping the initial report (Matthew and Barron, 2015; Seddon et al., 2012; Etherington, 2009), and presenting the research in a public forum (Etherington, 2005).

Studies using narrative approaches were given some acknowledgement if their recommendations were grounded in, and gave authority to, the experiences and needs vocalised by participating survivors (Byrne et al., 2017; Bond et al., 2018). Higher scores were allocated if researchers actively solicited the desired research impact from survivors, and if the policy and practice recommendations voiced by survivors were central to the report (Etherington, 2005, 2009; Seddon et al., 2012; Fisher et al., 2018; Lumbasi and Barron, 2016; Matthew and Barron, 2015; One in Four, 2015, 2019; Smith et al., 2015; IICSA, 2017; WYS and Fraser, 2006). However, only one study coupled survivors' involvement in developing recommendations with an impact evaluation plan that would also involve survivors (Seddon et al., 2012).

Beyond the broader impacts on policy and practice, studies were found to solicit the personal impact of involvement from participating survivors (Allnock and Miller, 2013; Seddon et al., 2012), and often evaluated and reported the benefits to advise future research practices with survivors (Etherington, 2005, 2009; Matthew and Barron, 2015; One in Four, 2015; IICSA, 2017). Survivor-led narrative approaches to data collection were frequently reported to have a therapeutic impact by providing a validating space for survivors to revisit, reflect and reinterpret past experiences (IICSA, 2017; One in Four, 2019). Furthermore, having one's voice listened to with the intent of reaching a wider audience was found to provide participating survivors with an empowering avenue to recognise the positive influence they can have on informing service provision and policy (Seddon et al., 2012; Etherington, 2009), and the lives of other survivors (IICSA, 2017; Lumbasi and Barron, 2016; One in Four, 2015, 2019). Among sources that consulted with survivors in project reference groups, only one study reported an outcome, which was participants being comforted by the presence of peers in the research (Moore et al., 2017).

In research conducted by survivors, benefits of increased self-awareness and reduced isolation arose for survivor-researchers as a result of analysing participant accounts and validating the impact of their own trauma in relation to others (Matthew and Barron, 2015; Etherington, 2005, 2009). Additionally, participants of studies involving survivor-researchers reported a decreased sense of isolation by being a part of a research community of survivors (Matthew and Barron, 2015; Etherington, 2005, 2009). Last, when participants conducted aspects of research authored by survivors, they were found to have challenged their preconceived abilities, increased self-confidence and, for some, experienced transformative changes by pursuing career and academic opportunities (Matthew and Barron, 2015).

\section{Accessibility}

Grey literature reports were found to be widely accessible through organisation or agency websites, and most published sources were available through open access. However, the four published studies 
conducted by survivor-researchers had restricted access in peer-reviewed journals only available through institutional membership or fees. Furthermore, these studies had a primary (Etherington, 2009; Fisher et al., 2018) or secondary purpose (Matthew and Barron, 2015; Etherington, 2005) of advocating for survivors' involvement to an academic audience.

\section{Limitations}

Insufficient reporting of involvement practices potentially introduced misclassification errors if the review excluded studies that failed to report practices. With the exception of research conducted by survivors, reporting of involvement practices was found to be higher in grey literature research. However, omission of information in research published in peer-reviewed journals may reflect restrictive publishing conditions, rather than a lack of, or limited, survivor involvement (Staley, 2009). As a result, involvement scores may be biased in favour of grey literature sources if authors were at greater liberty to discuss such practices.

A particular limitation of the ladder itself, and the model in general, is the lack of recognition given to involvement practices that seek to engage diverse and hard-to-reach groups of survivors, and which accommodate varying or flexible degrees of involvement. Placing value on research offering opportunities for high-quality involvement without giving recognition to low-level involvement opportunities could present issues of being exclusionary towards willing individuals who feel unable to adopt leadership or co-producer roles. Additionally, the evaluation of involvement did not assess the potential value of involvement itself progressing organically 'up the ladder', with survivors negotiating their own involvement as their relationships to the research and researchers grow (Shimmin et al., 2017).

These potential issues reflect past criticisms of the ladder model for limiting involvement to a onedimensional and static transfer of a finite amount of power (Funk et al., 2012; Tritter and McCallum, 2006). Alternatively, a scaffolding model has been favoured for its ability to recognise diversity in terms of backgrounds, abilities, interests and availability that may impact the degree of involvement that is desired by individuals. Future users of the ladder may address these issues by giving multiple scores under research activities if multiple roles are reported.

\section{Discussion}

\section{Extent of involvement}

There is an overall lack of involvement with survivors in the UK's evidence base. This review found that involvement with survivors is in its relative infancy, as only 12.9 per cent of the research 'for', 'about' or 'to' survivors was found to be conducted 'by' or 'with' survivors, compared with 62.5 per cent of primary care research in mental health reporting involvement (Blackburn et al., 2018). Some have argued that a lack of reporting may reflect an absence of activities or deliberate omission of low-quality practices (Price et al., 2018), but well-documented issues of reporting PPI suggest that these findings cannot be definitively attributed to a lack of involvement with survivors in practice.

However, if low reporting influenced the results, this presents its own concerns. First, it dilutes the evidence base and hinders advancements in establishing best practices for PPI with survivors. Additionally, service providers or policymakers seeking to draw from evidence may lack critical pieces of information for successful implementation (Beckett et al., 2018). Insufficient reporting also fails to set the precedent that involving survivors should be standard, normalised and widespread in research (Hughes and Duffy, 2018).

\section{Quality of involvement}

Involvement practices with survivors primarily reflect the current state of PPI practices in the UK, with an observed tendency to follow tokenistic models of low-quality involvement (Beresford, 2002). However, the 
absence of these models in priority-setting and planning stages suggests that current practices are failing to keep pace with mainstream developments (Green, 2016). Limiting involvement to an advisory capacity on predetermined approaches and priorities demonstrates a lack of commitment to, or awareness of, the benefits of genuine forms of involvement. Without deliberate and democratic spaces to centralise survivors' voices in shaping agendas, research critically misses the innovative potential gained from experiential knowledge (Beresford, 2005; Faulkner, 2017). Furthermore, pragmatically motivated practices to improve the acceptability and credibility of research consequently reinforce the status and power of professional knowledge (Green and Johns, 2019), thus further marginalising the voices of survivors.

When involvement was motivated by researchers seeking to empower the voices of participating survivors, collaborative approaches to data collection demonstrated the value of involvement beyond the pragmatic benefits of increased validity and reliability. Therapeutic elements of having one's experiences heard, valued and shared emerged from these approaches (Nurser et al., 2018). Notably, these practices should be regarded as being distinct from Arnstein's (1969) non-participatory 'Therapy' rung. Under this type of non-participation, the focus of engagement is on eradicating the subjects' 'pathology', rather than on changing the social conditions that produce and reinforce their 'pathologies' (Arnstein, 1969). Conversely, providing a space for survivors to have and control their voice disrupts the dominant culture of silence and stigma surrounding child abuse. It is noteworthy that such practices are not inherently emancipatory. Alcoff and Grey (1993) have warned against the transgressive potential of researchers coopting and defining survivors' voices and presenting them within hegemonic structures. Some researchers have also warned of survivors feeling pressure to conform to the research agenda, and the potential for social desirability bias to influence narratives (Gibson and Morgan, 2013; Campbell et al., 2010).

Resembling the mental health survivor-research movement (Faulkner, 2017; Sweeney, 2016), the need for the current research culture to embrace democratic and emancipatory approaches has been championed by survivors advocating for involvement in academic spaces. Survivor-researchers have rejected the exclusionary norms of 'objectivity' and 'neutrality' by producing knowledge situated in personal experiences and reflective of collective priorities (Rose, 2017). As a result, the research conducted by survivors has presented the practical model and theoretical basis for the level and quality of involvement that is desired and needed to produce a survivor-informed evidence base (Halliday et al., 2003).

\section{Recommendations}

\section{Implications for future research}

In addition to an increased need for involvement, research practices should seek to harness the experiential knowledge of survivors and to provide opportunities to realise the transformative impact of involvement on policy and practice. Research drawing from the expertise of survivors should not limit contributions to a preconceived notion that abuse experiences constitute the overwhelming extent of survivor identities. Instead, research should promote multilayered identities and abilities by providing survivors with opportunities to contribute, and to be financially compensated for their knowledge, skills and expertise beyond experiences of abuse (Lambert and Carr, 2018). Researchers should accommodate a range of involvement opportunities, and give survivors a high degree of control in shaping, negotiating and evolving their roles by continually revisiting the level of survivors' involvement throughout the research process. Researchers should seek to avoid 'box-ticking' practices, by involving survivors at the early conceptualisation of studies, in defining priority questions to be addressed, in shaping grant applications and study designs, empowering each individual participating in research, and extending involvement to subjects/participants by ensuring survivors' voices lead data collection and analysis.

Categorisations used in research to conceptualise abuse have been recognised by survivors as oversimplistic and ineffective in capturing complexity in real-life experience (Robotham et al., 2019). The under-representation of certain groups, and the limited attention given to intra-group differences in research, presents challenges for developing policies and practices that can appropriately and 
effectively respond to the multidimensional and complex needs of survivors. Therefore, active steps need to be taken to ensure that diverse backgrounds and identities are well represented and included in research. Integrating survivor involvement in recruitment strategies presents an invaluable opportunity to develop appropriate, sensitive and creative engagement (Martin, 2008). It also presents a critical means of addressing barriers of distrust, stigma and low awareness that result in the under-representation of disadvantaged groups in research (Bonevski et al., 2014), whose engagement in research activities could be facilitated and supported through outreach and recruitment strategies that are meaningful and driven by the values that underpin survivor involvement in research (Gillard et al., 2017; Russo, 2012).

Further research is needed to capture and evaluate the range of impacts, and ascertain the value of survivor involvement for research, policy and practice. These advancements are, however, limited by what is reported by researchers and what subsequently constitutes the evidence base covering processes, practices and outcomes of survivor involvement. Current reporting issues and insufficient documentation of involvement activities suggest that there is a need to make reporting on survivor involvement expected and required as standard practice by funding organisations, ethics committees and external reviewers and observers. This critical shift in the academic reporting culture may be further prompted by expanding opportunities for involvement in contributing to manuscripts and presenting research to academic audiences to increase the visibility, acceptability and credibility of survivor-researchers in the field. In addition to improving the richness and depth of reporting on the processes and outcomes of survivor involvement, research should seek and share the perspectives from all survivors involved to further understand and conceptualise successful involvement.

\section{Recommended adaptations to the Survivor Research Involvement Ladder}

The review found that the Survivor Research Involvement Ladder (Chevous et al., 2019) was effective for conducting a critical and comparative evaluation of survivor involvement. However, the tool did not assess some critical research areas, namely evaluating the breadth of approaches to data collection and validating the observed benefit of narrative approaches. With the addition of a 'Data collection' column, assessments of collaborative survivor-led approaches to data collection should be categorised as a coproduced activity. Conversely, if a survey is designed without input from survivors, this should be classified as survivors being subjects or participants of the research.

In future replication studies, the tool should validate research seeking and involving a diverse range of survivor voices due to the under-representation of certain groups within the selected studies. Under an additional 'Recruitment' column, value should be given to researchers adopting open and fair approaches to recruiting survivors as researchers or participants. Studies should also be assessed for addressing potential barriers to involvement and placing value on unconventional methods developed by or with survivors. Higher-quality involvement should be recognised if survivors lead or co-produce recruitment strategies and materials. When considering these recommendations, good involvement practices must continue to be respectful of confidentiality, maintain safety and engage in sensitive recruitment activities.

Last, the tool should recognise research that seeks to be flexible to the interests of survivors by providing a breadth of opportunities for involvement. This may be integrated into evaluations under the 'Planning and decision making' column by giving value to research that recognises and plans to accommodate different degrees of involvement based on survivors' interests. To validate these activities in practice, use of the tool should be flexible and user-led, rather than hierarchical and prescriptive, and resemble a scaffolding model to accommodate scoring of multiple roles if observed in a single research activity.

\section{Summary}

This review finds considerable opportunity for improvements in the extent and quality of involvement with survivors of childhood abuse in the UK's evidence base. With these findings and the introduction of the 
Survivor Research Involvement Ladder (Chevous et al., 2019), we hope that others will be encouraged to give space and authority to survivors' voices through meaningful and genuine engagement.

The ladder is currently in its pilot phase, and it is available to download (https://survivorsvoices. org/involvement-ladder/). Survivors Voices is gathering feedback to evaluate and maximise the ladder's effectiveness in creating research that is genuinely co-produced with people who have experienced abuse.

\section{Acknowledgements}

The researchers would like to thank Russell Burke (London School of Hygiene \& Tropical Medicine) for assistance in developing the search strategy.

\section{Declarations and conflicts of interest}

\section{Conflicts of interest}

The authors declare no conflicts of interest with this work. All efforts to sufficiently anonymise the author during peer review of this article have been made. The authors declare no further conflicts with this article.

\section{References}

Adams, J., Hillier-Brown, F.C., Moore, H.J., Lake, A.A., Araujo-Soares, V., White, M. and Summerbell, C. (2016) 'Searching and synthesising "grey literature" and "grey information" in public health: Critical reflections on three case studies'. Systematic Reviews, 5 (1), Article 164, 1-11. https://doi.org/10.1186/s13643-016-0337-y.

Alcoff, L. and Gray, L. (1993) 'Survivor discourse: Transgression or recuperation?' Signs: Journal of Women in Culture and Society, 18 (2), 260-90. https://doi.org/10.1086/494793.

Allnock, D. and Miller, P. (2013) No One Noticed, No One Heard: A study of disclosures of childhood abuse. London: NSPCC. Accessed 10 September 2021. https://learning.nspcc.org.uk/media/1052/no-one-noticed-no-one-heard-report.pdf.

Anderson, K.M. and Hiersteiner, C. (2008) 'Recovering from childhood sexual abuse: Is a "storybook ending" possible?' The American Journal of Family Therapy, 36 (5), 413-24. https://doi.org/10.1080/01926180701804592.

Arnstein, S.R. (1969) 'A ladder of citizen participation'. Journal of the American Planning Association, 35 (4), $216-24$. https://doi.org/10.1080/01944366908977225.

Beckett, K., Farr, M., Kothari, A., Wye, L. and Le May, A. (2018) 'Embracing complexity and uncertainty to create impact: Exploring the processes and transformative potential of co-produced research through development of a social impact model'. Health Research Policy and Systems, 16 (1), Article 118, 1-19. https://doi.org/10.1186/s12961-018-0375-0.

Beresford, P. (2002) 'User involvement in research and evaluation: Liberation or regulation?'. Social Policy and Society, 1 (2), 95-105. https://doi.org/10.1017/s1474746402000222.

Beresford, P. (2005) 'Developing the theoretical basis for service user/survivor-led research and equal involvement in research'. Epidemiologia e Psichiatria Sociale, 14 (1), 4-9. https://doi.org/10.1017/S1121189X0000186X.

Beresford, P. (2013a) 'From "other" to involved: User involvement in research: An emerging paradigm'. Nordic Social Work Research, 3 (2), 139-48. https://doi.org/10.1080/2156857x.2013.835138.

Beresford, P. (2013b) Beyond the Usual Subjects: Towards inclusive user involvement. London: Shaping Our Lives. Accessed 10 September 2021. https://shapingourlives.org.uk/report/beyond-the-usual-suspects-findings/.

Blackburn, S., McLachlan, S., Jowett, S., Kinghorn, P., Gill, P., Higginbottom, A., Rhodes, C., Stevenson, F. and Jinks, C. (2018) 'The extent, quality and impact of patient and public involvement in primary care research: A mixed methods study'. Research Involvement and Engagement, 4 (1), Article 16, 1-18. https://doi.org/10.1186/s40900-018-0100-8.

Bond, E., Ellis, F. and McCusker, J. (2018) I'll Be a Survivor for the Rest of My Life: Adult survivors of child sexual abuse and their experience of support services. Ipswich: University of Suffolk and Survivors in Transition. Accessed 10 September 2021. https://www.basw.co.uk/system/files/resources/\%E2\%80\%981\%E2\%80\%99||\%20be\%20a\%20survivor\%20for\%20 the\%20rest\%20of\%20my\%20life\%E2\%80\%99\%20Report\%20FINAL.pdf.

Bonevski, B., Randell, M., Paul, C., Chapman, K., Twyman, L., Bryant, J., Brozek, I. and Hughes, C. (2014) 'Reaching the hard-to-reach: A systematic review of strategies for improving health and medical research with socially disadvantaged groups'. BMC Medical Research Methodology, 14 (1), Article 42, 1-29. https://doi.org/10.1186/1471-2288-14-42.

Brett, J., Staniszewska, S., Mockford, C., Herron-Marx, S., Hughes, J., Tysall, C. and Suleman, R. (2014) 'A systematic review of the impact of patient and public involvement on service users, researchers and communities'. Patient, 7 (4), 387-95. https://doi.org/10.1007/s40271-014-0065-0. 
Byrne, J., Smart, C. and Watson, G. (2017) '"I felt like I was being abused all over again": How survivors of child sexual abuse make sense of the perinatal period through their narratives'. Journal of Child Sexual Abuse, 26 (4), $465-86$. https://doi.org/10.1080/10538712.2017.1297880.

Campbell, R., Adams, A.E., Wasco, S.M., Ahrens, C.E. and Sefl, T. (2010) '“What has it been like for you to talk with me today?": The impact of participating in interview research on rape survivors'. Violence Against Women, 16 (1), 60-83. https://doi.org/10.1177/1077801209353576.

Carr, A., Duff, H. and Craddock, F. (2018) 'A systematic review of reviews of the outcome of noninstitutional child maltreatment'. Trauma, Violence, and Abuse, 21 (4), 828-43. https://doi.org/10.1177/1524838018801334.

Chevous, J., Bewley, S., Kennedy, S. and Perôt, C. (2019) 'Survivors Voices: Survivor involvement in research ladder (pilot draft 2)'. Accessed 12 September 2021. https://survivorsvoices.org/involvement-ladder/.

Chouliara, Z., Karatzias, T., Scott-Brien, G., Macdonald, A., MacArthur, J. and Frazer, N. (2012) 'Adult survivors' of childhood sexual abuse perspectives of services: A systematic review'. Counselling \& Psychotherapy Research, 12 (2), 146-61. https://doi.org/10.1080/14733145.2012.656136.

Crepaz-Keay, D. (2016) 'Can we co-produce reality, normality, and a world that has meaning for all?'. Philosophy, Psychiatry, \& Psychology, 23 (3/4), 313-21. https://doi.org/10.1353/ppp.2016.0037.

Crompton, A. (2019) 'Inside co-production: Stakeholder meaning and situated practice'. Social Policy and Administration, 53 (2), 219-32. https://doi.org/10.1111/spol.12466.

Dorrepaal, E., Thomaes, K., Hoogendoorn, A.W., Veltman, D.J., Draijer, N. and Van Balkom, A.J. (2014) 'Evidence-based treatment for adult women with child abuse-related complex PTSD: A quantitative review'. European Journal of Psychotraumatology, 5, Article 23613, 1-18. https://doi.org/10.3402/ejpt.v5.23613.

Ehring, T., Welboren, R., Morina, N., Wicherts, J.M., Freitag, J. and Emmelkamp, P.M.G. (2014) 'Meta-analysis of psychological treatments for posttraumatic stress disorder in adult survivors of childhood abuse'. Clinical Psychology Review, 34 (8), 645-57. https://doi.org/10.1016/j.cpr.2014.10.004.

Elliott, J., Horwood, A., Hunn, A., Staley, K. and Tarpey, M. (2017) Public Involvement in Applications to the Health Research Authority Research Ethics Service: Comparative analysis of data from 2010, 2012 and 2014. London: HRA London and INVOLVE Southampton. Accessed 10 September 2021. https://s3.eu-west-2.amazonaws.com/www.hra. nhs.uk/media/documents/public-involvement-applications-hra.pdf.

Etherington, K. (2005) 'Researching trauma, the body and transformation: A situated account of creating safety in unsafe places'. British Journal of Guidance and Counselling, 33 (3), 299-314. https://doi.org/10.1080/03069880500179400.

Etherington, K. (2009) 'Life story research: A relevant methodology for counsellors and psychotherapists'. Counselling and Psychotherapy Research, 9 (4), 225-33. https://doi.org/10.1080/14733140902975282.

Faulkner, A. (2004) The Ethics of Survivor Research: Guidelines for the ethical conduct of research carried out by mental health service users and survivors. Bristol: The Policy Press. Accessed 10 September 2021. https://www.jrf.org.uk/ report/ethics-survivor-research-guidelines-ethical-conduct-research-carried-out-mental-health.

Faulkner, A. (2017) 'Survivor research and Mad Studies: The role and value of experiential knowledge in mental health research'. Disability and Society, 32 (4), 500-20. https://doi.org/10.1080/09687599.2017.1302320.

Felitti, M.D., Vincent, J., Anda, M.D., Robert, F., Nordenberg, M.D., Williamson, M.S., David, F., Spitz, M.S., Alison, M. and Edwards, B.A. (1998) 'Relationship of childhood abuse and household dysfunction to many of the leading causes of death in adults: The Adverse Childhood Experiences (ACE) Study'. American Journal of Preventive Medicine, 14 (4), 245-58. https://doi.org/10.1016/S0749-3797(98)00017-8.

Fisher, P., Balfour, B. and Moss, S. (2018) 'Advocating co-productive engagement with marginalised people: A specific perspective on and by survivors of childhood sexual abuse'. British Journal of Social Work, 48 (7), $2096-113$. https://doi.org/10.1093/bjsw/bcx149.

Funk, A., Van Borek, N., Taylor, D., Grewal, P., Tzemis, D. and Buxton, J.A. (2012) 'Climbing the "Ladder of Participation": Engaging experiential youth in a participatory research project'. Canadian Journal of Public Health, 103 (4), $288-92$. https://doi.org/10.1007/bf03404237.

Gibson, K. and Morgan, M. (2013) 'Narrative research on child sexual abuse: Addressing perennial problems in quantitative research'. Qualitative Research in Psychology, 10 (3), 298-317. https://doi.org/10.1080/14780887.2011.606597.

Gillard, S., Foster, R., Gibson, S., Goldsmith, L., Marks, J. and White, S. (2017) 'Describing a principles-based approach to developing and evaluating peer worker roles as peer support moves into mainstream mental health services'. Mental Health and Social Inclusion, 21 (3), 133-43. https://doi.org/10.1108/MHSI-03-2017-0016.

Godin, K., Stapleton, J., Kirkpatrick, S.I., Hanning, R.M. and Leatherdale, S.T. (2015) 'Applying systematic review search methods to the grey literature: A case study examining guidelines for school-based breakfast programs in Canada'. Systematic Reviews, 4 (1), Article 138, 1-10. https://doi.org/10.1186/s13643-015-0125-0.

Green, G. (2016) 'Power to the people: To what extent has public involvement in applied health research achieved this?'. Research Involvement and Engagement, 2 (1), 1-13. https://doi.org/10.1186/s40900-016-0042-y.

Green, G. and Johns, T. (2019) 'Exploring the relationship (and power dynamic) between researchers and public partners working together in applied health research teams'. Frontiers in Sociology, 4, Article 20, 1-10. https://doi.org/10.3389/ fsoc.2019.00020. 
Halliday, M., Sherwood, L. and Faulkner, A. (2003) 'Mental health user/survivor research in the UK'. Updates, 5 (2), 1-6. Accessed 10 September 2021. http://studymore.org.uk/mhf1103.pdf.

Hart, R. (1992) Children's Participation: From tokenism to citizenship. Florence: UNICEF International Child Development Centre. Accessed 10 September 2021. https://www.unicef-irc.org/publications/pdf/childrens_participation.pdf.

Holland-Hart, D.M., Addis, S.M., Edwards, A., Kenkre, J.E. and Wood, F. (2019) 'Coproduction and health: Public and clinicians' perceptions of the barriers and facilitators'. Health Expectations, 22 (1), 93-101. https://doi.org/10.1111/ hex.12834.

Hong, Q.N., Pluye, P., Fàbregues, S., Bartlett, G., Boardman, F., Cargo, M., Dagenais, P., Gagnon, M.P., Griffiths, F., Nicolau, B., Rousseau, M.P. and Vedel, I. (2018) Mixed Methods Appraisal Tool (MMAT), Version 2018. Accessed 10 September 2021. http://mixedmethodsappraisaltoolpublic.pbworks.com/w/file/fetch/127916259/MMAT_2018_criteriamanual_2018-08-01_ENG.pdf.

Hughes, M. and Duffy, C. (2018) 'Public involvement in health and social sciences research: A concept analysis'. Health Expectations, 21 (6), 1183-190. https://doi.org/10.1111/hex.12825.

IICSA (Independent Inquiry into Child Sexual Abuse) (2017) Victim and Survivor Voices from The Truth Project. Accessed 10 September 2021. https://www.iicsa.org.uk/key-documents/3304/view/victim-survivor-voices-from-truth-project.pdf.

Jennings, H., Slade, M., Bates, P., Munday, E. and Toney, R. (2018) 'Best practice framework for Patient and Public Involvement (PPI) in collaborative data analysis of qualitative mental health research: Methodology development and refinement'. BMC Psychiatry, 18 (1), Article 213, 1-14. https://doi.org/10.1186/s12888-018-1794-8.

Kalathil, J. and Jones, N. (2016) 'Unsettling disciplines: Madness, identity, research, knowledge'. Philosophy, Psychiatry and Psychology, 23 (3/4), 183-8. https://doi.org/10.1353/ppp.2016.0016.

Kara, H. (2013) 'Mental health service user involvement in research: Where have we come from, where are we going?'. Journal of Public Mental Health, 12 (3), 122-35. https://doi.org/10.1108/JPMH-01-2013-0001.

LaFrance, D. (2017) 'Finding my voice: Surviving childhood sexual abuse'. Electronic Theses and Dissertations, 1682. Accessed 10 September 2021. https://digitalcommons.georgiasouthern.edu/cgi/viewcontent.cgi?article=2805\&context=etd.

Lambert, N. and Carr, S. (2018) '"Outside the original remit": Co-production in UK mental health research, lessons from the field'. International Journal of Mental Health Nursing, 27 (4), 1273-81. https://doi.org/10.1111/inm.12499.

Lefebvre, C., Glanville, J., Briscoe, S., Littlewood, A., Marshall, C., Metzendorf, M.-I., Noel-Storr, A., Rader, T., Shokraneh, F., Thomas, J. and Wieland, L.S. (2011) 'Chapter 4: Searching for and selecting studies'. In J. Higgins, J. Thomas, J. Chandler, M. Cumpston, T. Li, M.J. Page and V.A. Welch (eds), Cochrane Handbook for Systematic Reviews of Interventions, Version 6.1 (updated September 2020). The Cochrane Collaboration. Accessed 13 December 2021. https://training.cochrane.org/handbook/archive/v6.1/chapter-04\#section-4-1.

Lumbasi, J. and Barron, I. (2016) 'Male survivor perceptions of seeking support: Hermeneutic analysis of a cluster case study'. International Journal of Social Work, 3 (1), 91-105. https://doi.org/10.5296/ijsw.v3i1.8626.

MacGinley, M., Breckenridge, J. and Mowll, J. (2019) 'A scoping review of adult survivors' experiences of shame following sexual abuse in childhood'. Health and Social Care in the Community, 27, 1135-46. https://doi.org/10.1111/hsc.12771.

Martin, G.P. (2008) '"Ordinary people only": Knowledge, representativeness, and the publics of public participation in healthcare'. Sociology of Health and Illness, 30 (1), 35-54. https://doi.org/10.1111/j.1467-9566.2007.01027.x.

Mathie, E., Wilson, P., Poland, F., McNeilly, E., Howe, A., Staniszewska, S., Cowe, M., Munday, D. and Goodman, C. (2014) 'Consumer involvement in health research: A UK scoping and survey'. International Journal of Consumer Studies, 38 (1), 35-44. https://doi.org/10.1111/ijcs.12072.

Matthew, L. and Barron, I.G. (2015) 'Participatory action research on help-seeking behaviors of self-defined ritual abuse survivors: A brief report'. Journal of Child Sexual Abuse, 24 (4), 429-43. https://doi.org/10.1080/10538712.2015.1029104.

Mays, N., Pope, C. and Popay, J. (2005) 'Systematically reviewing qualitative and quantitative evidence to inform management and policy-making in the health field'. Journal of Health Services Research and Policy, 10 (Suppl 1), 6-20. https://doi.org/10.1258/1355819054308576.

McClain, N. and Amar, A.F. (2013) 'Female survivors of child sexual abuse: Finding voice through research participation'. Issues in Mental Health Nursing, 34 (7), 482-7. https://doi.org/10.3109/01612840.2013.773110.

Moore, J., Thornton, C. and Hughes, M. (2017) 'On the road to resilience: The help-seeking experiences of Irish emigrant survivors of institutional abuse'. Child Abuse Review, 26 (5), 375-87. https://doi.org/10.1002/car.2415.

Munro, I. and Randall, J. (2007) '"I don't know what I'm doing, how about you?": Discourse and identity in practitioners dealing with the survivors of childhood sexual abuse'. Organization, 14 (6), 887-907. https://doi.org/10.1177/1350508407085415.

Nurser, K.P., Rushworth, I., Shakespeare, T. and Williams, D. (2018) 'Personal storytelling in mental health recovery'. Mental Health Review Journal, 23 (1), 25-36. https://doi.org/10.1108/MHRJ-08-2017-0034.

One in Four (2015) Survivors' Voices: Breaking the silence on living with the impact of child sexual abuse in the family environment. London: One in Four. Accessed 10 September 2021. https://oneinfour.org.uk/wp-content/ uploads/2015/11/Survivors_Voices_Report_November_2015-2.pdf.

One in Four (2019) Numbing the Pain: Survivors' voices of childhood sexual abuse and addiction. London: One in Four. Accessed 10 September 2021. https://oneinfour.org.uk/wp-content/uploads/2019/03/RackMultipart20190320-8959khxw2x.pdf. 
Parry, S. and Simpson, J. (2016) 'How do adult survivors of childhood sexual abuse experience formally delivered talking therapy?: A systematic review'. Journal of Child Sexual Abuse, 25 (7), 793-812. https://doi.org/10.1080/10538712.2016. 1208704.

Paylor, J. and McKevitt, C. (2019) 'The possibilities and limits of "co-producing" research'. Frontiers in Sociology, 4, Article 23, 1-5. https://doi.org/10.3389/fsoc.2019.00023.

Perôt, C., Chevous, J. and Survivors Voices Research Group (2018) Turning Pain into Power: A charter for organisations engaging abuse survivors in projects, research \& service development. Accessed 10 September 2021. http://survivorsvoices.org/wp-content/uploads/2018/10/Survivors-Charter-Final-V1-for-Piloting-Oct-2018.pdf.

Pluye, P., Hong, Q.N., Robert, E., Cargo, M., Bartlett, G., O'Cathain, A., Griffiths, F., Boardman, F., Gagnon, M.P. and Rousseau, M.C. (2011) Proposal: A mixed methods appraisal tool for systematic mixed studies reviews. Montreal: Department of Family Medicine, McGill University. Accessed 10 September 2021. http://mixedmethodsappraisaltoolpublic.pbworks. com/w/file/fetch/84371689/MMAT\%202011\%20criteria\%20and\%20tutorial\%202011-06-29updated2014.08.21.pdf.

Price, A., Schroter, S., Snow, R., Hicks, M., Harmston, R., Staniszewska, S., Parker, S. and Richards, T. (2018) 'Frequency of reporting on patient and public involvement (PPI) in research studies published in a general medical journal: A descriptive study'. BMJ Open, 8 (3), Article e020452, 1-10. https://doi.org/10.1136/bmjopen-2017-020452.

Radhika, K., Manjula, M. and Jaisoorya, T.S. (2018) 'Ethical gaps in conducting research among adult survivors of child sexual abuse: A review'. Indian Journal of Medical Ethics, 3 (3), 186-92. https://doi.org/10.20529/IJME.2018.046.

Ramirez, L.A. and Allison, V.A. (2018) 'Breaking the silence surrounding childhood sexual abuse: Consequences for our practice and selves as teacher educators'. Reflective Practice, 19 (4), 447-60. https://doi.org/10.1080/14623943.2018.14 79692.

Robotham, D., Sweeney, A. and Perôt, C. (2019) Survivors' Priority Themes and Questions for Research. London: Violence Abuse and Mental Health Network. Accessed 10 September 2021. https://www.vamhn.co.uk/uploads/1/2/2/7/122741688/ consultation_report_on_website.pdf.

Rose, D. (2017) 'Service user/survivor-led research in mental health: Epistemological possibilities'. Disability and Society, 32 (6), 773-89. https://doi.org/10.1080/09687599.2017.1320270.

Russo, J. (2012) 'Survivor-controlled research: A new foundation for thinking about psychiatry and mental health'. Forum: Qualitative Social Research, 13 (1), Article 8. https://doi.org/10.17169/fqs-13.1.1790.

Seddon, D., Krayer, A., Robinson, C., Gwilym, H., McKeown, G. and Hodrien, C. (2012) Adult Survivors and Their Families: Current needs and service responses. Bangor: National Institute for Social Care and Health Research. Accessed 10 September 2021. https://cfmhas.org.uk/wp-content/uploads/2020/03/Adult-survivors-and-their-families-finalreport-17-01-12.pdf.

Shimmin, C., Wittmeier, K.D.M., Lavoie, J.G., Wicklund, E.D. and Sibley, K.M. (2017) 'Moving towards a more inclusive patient and public involvement in health research paradigm: The incorporation of a trauma-informed intersectional analysis'. BMC Health Services Research, 17, Article 539, 1-10. https://doi.org/10.1186/s12913-017-2463-1.

Smith, N., Dogaru, C. and Ellis, F. (2015) Hear Me. Believe Me. Respect Me: A survey of adult survivors of child sexual abuse and their experiences of support services. Ipswich: University Campus Suffolk. Accessed 10 September 2021. https://www.basw.co.uk/system/files/resources/basw_122305-1_0.pdf.

Staley, K. (2009) Exploring Impact: Public involvement in NHS, public health and social care research. Eastleigh: INVOLVE. Accessed 10 September 2021. https://www.invo.org.uk/wp-content/uploads/2011/11//nvolve_Exploring_ Impactfinal28.10.09.pdf.

Sweeney, A. (2016) 'Why Mad Studies needs survivor research and survivor research needs Mad Studies'. Intersectionalities: A Global Journal of Social Work Analysis, Research, Polity, and Practice, 5 (3), 36-61. Accessed 10 September 2021. https://journals. library.mun.ca/ojs/index.php/IJ/article/view/1614/1330.

Sweeney, A., Beresford, P., Faulkner, A., Nettle, M. and Diana, R. (eds) (2009) This is Survivor Research. Ross-on-Wye: PCCS Books.

Tambuyzer, E., Pieters, G. and Van Audenhove, C. (2014) 'Patient involvement in mental health care: One size does not fit all'. Health Expectations, 17 (1), 138-50. https://doi.org/10.1111/j.1369-7625.2011.00743.x.

Tembo, D., Morrow, E., Worswick, L. and Lennard, D. (2019) 'Is co-production just a pipe dream for applied health research commissioning?: An exploratory literature review'. Frontiers in Sociology, 4, Article 50, 1-12. https://doi.org/10.3389/fsoc.2019.00050.

Thompson, J., Bissell, P., Cooper, C., Armitage, C.J. and Barber, R. (2012) 'Credibility and the "professionalized" lay expert: Reflections on the dilemmas and opportunities of public involvement in health research'. Health: An Interdisciplinary Journal for the Social Study of Health, Illness and Medicine, 16 (6), 602-18. https://doi.org/10.1177/1363459312441008.

Thompson, J., Bissell, P., Cooper, C.L., Armitage, C.J. and Barber, R. (2014) 'Exploring the impact of patient and public involvement in a cancer research setting'. Qualitative Health Research, 24 (1), $46-54$. https://doi.org/10.1177/1049732313514482.

Tritter, J.Q. and McCallum, A. (2006) 'The snakes and ladders of user involvement: Moving beyond Arnstein'. Health Policy, 76 (2), 156-68. https://doi.org/10.1016/j.healthpol.2005.05.008. 
Urbanowicz, C. and Reinke, B.A. (2018) 'Publication overlap: Building an academic house with salami shingles'.

The Bulletin of the Ecological Society of America, 99 (4), Article e01435, 1-6. https://doi.org/10.1002/bes2.1425.

Von Elm, E., Poglia, G., Walder, B. and Tramèr, M.R. (2004) 'Different patterns of duplicate publication: An analysis of articles used in systematic reviews'. JAMA, 291 (8), 974-80. https://doi.org/10.1001/jama.291.8.974.

Wright, C. and Gabriel, L. (2018) 'Perspectives of adult survivors of child sexual abuse: An exploration of the adjustments to self-structure through meaning-making in therapy'. Journal of Child Sexual Abuse, 27 (6), 663-81. https://doi.org/10.1080/10538712.2018.1496961.

WYS (West Yorkshire Survivors) and Fraser, C. (2006) A View From Inside the Box: A social research project exploring sexual abuse/violence service provision across the Bradford district. Bradford: Survivors West Yorkshire. Accessed 10 September 2021. https://survivorswestyorkshire.org.uk/download/a-view-from-inside-the-box-i/. 